\title{
Covariant Description of Composite Meson Systems and Chiral Symmetry
}

\author{
Shin Ishida, ${ }^{\mathrm{a}}$ Muneyuki Ishida ${ }^{\mathrm{b}}$ and Tomohito Maeda ${ }^{\mathrm{a}}$ \\ ${ }^{a}$ Coll. Sci. and Tech. Nihon U., Kanda-Surugadai, Chiyoda, Tokyo 101-0062, Japan \\ ${ }^{\mathrm{b}}$ Department of Physics, Tokyo Institute of Technology, Tokyo 152-8551, Japan
}

\begin{abstract}
Assuming the spin-independence for confining force, we give a covariant quark representation of general composite meson systems with definite Lorentz transformation properties. For benefit of this representation we are able to deduce automatically the transformation rules of composite mesons for general symmetry operations from those of constituent (exciton) quarks. Applying this we investigate especially physical implication of chiral symmetry for the meson systems, and point out a possibility of existence of new meson multiplets.
\end{abstract}

\section{Introduction}

There are the two contrasting view points of composite quark-antiquark mesons: The one is non-relativistic, based on the approximate symmetry of $L S$-coupling in NRQM; while the other is relativistic, based on the dynamically broken chiral symmetry in the NJL model. The $\pi$-meson (or $\pi$-nonet) is now widely believed to have a dual nature of non-relativistic particle with $(L, S)=(0,0)$ and also of relativistic particle as a NambuGoldston boson with $J^{P}=0^{-}$in the case of spontaneous breaking of chiral symmetry. However, no attempts to unify the above two view points have been yet proposed. On the other hand we have developed the covariant oscillator quark model (COQM) [1] for many years as a covariant extension of NRQM, which is based on the boosted $L S$-coupling scheme. The meson wave functions (WF) in COQM are tensors in the $\tilde{U}(4) \otimes O(3,1)$ space and reduce at the rest frame to those in the $S U(2)_{\text {spin }} \otimes O(3)_{\text {orbit }}$ space in NRQM. The COQM has been successful especially in treating the $Q \bar{Q}$ meson system and the $(q, \bar{Q})$ or $(Q, \bar{q})$ meson system, leading, respectively, to a satisfactory understanding of radiative transitions and to the same weak form factor relations as in HQET. However, in COQM no consideration on chiral symmetry has been given and it is not able to explain the dual nature of $\pi$ meson.

The purpose of the present talk is to get rid of this defect in COQM and is to give a unified view point of the two contrasting view points of the composite meson systems.

\section{Covariant Framework for Describing Composite Mesons}

For meson WF described by $\Phi_{A}^{B}\left(x_{1}, x_{2}\right)\left(x_{1}, x_{2}\right.$ denoting the space-time coordinate and $A=(\alpha, a)(B=(\beta, b))$ denoting the Dirac spinor and flavor indices of constituent quark 
(anti-quark)) we set up the bilocal Yukawa equation[2]

$\left[\partial^{2} / \partial X_{\mu}^{2}-\mathcal{M}^{2}\left(x_{\mu}, \partial / \partial x_{\mu}\right)\right] \Phi_{A}^{B}(X, x)=0$

( $X(x)$ denoting the center of mass (CM) (relative) coordinate of meson), where the $\mathcal{M}^{2}$ is squared mass operator including only a central, (Dirac) spinor

-independent confining potential. The WF is separated into the plane wave describing $\mathrm{CM}$ motion and the internal WF as

$\Phi_{A}^{B}\left(x_{1}, x_{2}\right)=\sum_{\mathbf{P}_{n}, n}\left(e^{i P_{n} X} \Psi_{n, A}^{(+) B}\left(x, P_{n}\right)+e^{-i P_{n} X} \Psi_{n, A}^{(-) B}\left(x, P_{n}\right)\right)$,

where $P_{n, \mu}^{2}=-M_{n}^{2}, P_{n, 0}=\sqrt{M_{n}^{2}+\mathbf{P}_{n}^{2}} ;( \pm)$ represents the positive (negative) frequency part; and $n$ does a freedom of excitation. We have the following field theoretical expression in mind for the WF and its Pauli-conjugate, $\bar{\Phi}_{B}{ }^{A}\left(x_{2}, x_{1}\right) \equiv\left[\gamma_{4} \Phi\left(x_{1}, x_{2}\right)^{\dagger} \gamma_{4}\right]_{B}{ }^{A}$ as

$\Phi_{A}^{B}\left(x_{1}, x_{2}\right)=\sum_{n}\left[\left\langle 0\left|\psi_{A}\left(x_{1}\right) \bar{\psi}^{B}\left(x_{2}\right)\right| M_{n}\right\rangle+\left\langle M_{n}\left|\psi_{A}\left(x_{1}\right) \bar{\psi}^{B}\left(x_{2}\right)\right| 0\right\rangle\right]$,

$\bar{\Phi}_{B}^{A}\left(x_{2}, x_{1}\right)=\sum_{n}\left[\left\langle M_{n}\left|\psi_{B}\left(x_{2}\right) \bar{\psi}^{A}\left(x_{1}\right)\right| 0\right\rangle+\left\langle 0\left|\psi_{B}\left(x_{2}\right) \bar{\psi}^{A}\left(x_{1}\right)\right| M_{n}\right\rangle\right]$,

where $\psi_{A}\left(\bar{\psi}^{B}\right)$ denotes the quark field (its Pauli-conjugate) and $\left|M_{n}\right\rangle$ does the composite meson state. The internal WF is, concerning the spin-dependence, expanded in terms of a complete set $\left\{W^{(i)}\right\}$ of free bi-Dirac spinors of quarks and anti-quarks; and the Fierzcomponent meson WF is expressed as

$\Psi_{A}^{( \pm) B}\left(x, P_{n}\right)=\sum_{(i)} W_{\alpha}^{(i)( \pm) \beta}\left(P_{n}\right) M_{a}^{(i)( \pm) b}\left(x, P_{n}\right), \quad M^{(i)( \pm)}\left(x, P_{n}\right)=\epsilon^{(i)}\left\langle\bar{W}^{(i)(\mp)} \Psi^{( \pm)}\right\rangle$,

where $\langle A\rangle$ means trace of $A$ and ortho-normal relations $\left\langle\bar{W}^{(i)(\mp)} W^{(j)( \pm)}\right\rangle=\epsilon^{(i)} \tilde{\delta}_{i j}\left(\epsilon^{(i)}\right.$ and $\tilde{\delta}_{i j}$ denote,respectively, the sign and pseudo-Kronecker symbols) is used.

The meson WF satisfies, as is seen from Eq.(3), the self-conjugate condition, leading to the (conventional) crossing rule (or substituion law) for the (composite) meson WF, as

$\bar{\Phi}_{A}^{B}\left(x_{1}, x_{2}\right)=\Phi_{A}^{B}\left(x_{1}, x_{2}\right), \quad \bar{M}_{b}^{(i)( \pm) a}\left(-x, P_{n}\right)=M_{a}^{(i)(\mp) b}\left(x, P_{n}\right)^{\dagger}=M_{b}^{(i)( \pm) a}\left(x, P_{n}\right)$.

\section{Complete Set of Spin Wave Function and Heavy-quark Meson Systems}

We set up the conventional "free" Dirac spinors with four-momentum of composite meson itself $P=P_{M}, D_{q, \alpha}(P) \equiv\left(u_{q, \alpha}^{(r)}(P), v_{q, \alpha}^{(r)}(P)(r=1,2)\right)$ for quarks and $\bar{D}_{\bar{q}}^{\beta}(P) \equiv$ $\left(\bar{v}_{\bar{q}}^{(s) \beta}(P), \bar{u}_{\bar{q}}^{(s) \beta}(P)(s=1,2)\right)$ for anti-quarks. It is to be noted that all four spinors are necessary for both quarks and anti-quarks inside of mesons. Then the complete set of bi-Dirac spinors is given by円

$$
\begin{aligned}
& \left\{W^{(i)(+)}(P)\right\} \quad: \quad U(P)=\left.u_{q}^{(r)}\left(p_{1}\right) \bar{v}_{\bar{q}}^{(s)}\left(p_{2}\right)\right|_{p_{i, \mu}=\kappa_{i} P_{\mu}}=u_{+}^{(r)}(\mathbf{P}) \bar{v}_{+}^{(s)}(\mathbf{P}), \\
& C(P)=\left.u_{q}^{(r)}\left(p_{1}\right) \bar{u}_{\bar{q}}^{(s)}\left(p_{2}\right)\right|_{p_{i, \mu}=\kappa_{i} P_{\mu}}=u_{+}^{(r)}(\mathbf{P}) \bar{v}_{-}^{(s)}(-\mathbf{P}), \\
& D(P)=\left.v_{q}^{(r)}\left(p_{1}\right) \bar{v}_{\bar{q}}^{(s)}\left(p_{2}\right)\right|_{p_{i, \mu}=\kappa_{i} P_{\mu}}=u_{-}^{(r)}(-\mathbf{P}) \bar{v}_{+}^{(s)}(\mathbf{P}), \\
& V(P)=\left.v_{q}^{(r)}\left(p_{1}\right) \bar{u}_{\bar{q}}^{(s)}\left(p_{2}\right)\right|_{p_{i, \mu}=\kappa_{i} P_{\mu}}=u_{-}^{(r)}(-\mathbf{P}) \bar{v}_{-}^{(s)}(-\mathbf{P}),
\end{aligned}
$$

\footnotetext{
1 In the following we give only the expressions of $(+)$-frequency parts, and consider only the ground states of composite system, disregarding the relative coordinates.
} 
where $u_{+}(\mathbf{P})\left(\bar{v}_{+}(\mathbf{P})\right)$ and $u_{-}(-\mathbf{P})\left(\bar{v}_{-}(-\mathbf{P})\right)$ denote the Dirac spinors with positive energy and momentum $\mathbf{P}$ and with negative energy and momentum $-\mathbf{P}$, respectively, describing quarks (anti-quarks). In Eq.(6) we have defined technically the momenta of constituent quarks (to be called quark-exciton) [3] as

$p_{i, \mu} \equiv \kappa_{i} P_{\mu}, p_{i, \mu}^{2}=-m_{i}^{2} ; P_{\mu}^{2}=-M^{2}, M=m_{1}+m_{2}\left(\kappa_{1,2} \equiv m_{1,2} /\left(m_{1}+m_{2}\right)\right)$.

The respective members in Eq.(6) satisfy a couple of the corresponding free Dirac equations in momentum space (which are equivalent to the (conventional or new-type of) Bargman-Wigner Equations) and are expressed in terms of their irreducible composite meson WF as follows:

$$
\begin{aligned}
& \text { (Non relat. comp.) } U_{A}^{B}(P)=\frac{1}{2 \sqrt{2}}\left[\left(i \gamma_{5} P_{s, a}^{(N R) b}(P)+i \gamma_{\mu} V_{\mu, a}^{(N R) b}(P)\right)\left(1+\frac{i P \gamma}{M}\right)\right]_{\alpha}{ }^{\beta}, \\
& \text { (Semi relat. comp.) } \quad C_{A}^{B}(P)=\frac{1}{2 \sqrt{2}}\left[\left(S_{a}^{(\bar{q}) b}(P)+i \gamma_{5} \gamma_{\mu} A_{\mu, a}^{(\bar{q}) b}(P)\right)\left(1-\frac{i P \gamma}{M}\right)\right]_{\alpha}{ }^{\beta}, \\
& D_{A}^{B}(P)=\frac{1}{2 \sqrt{2}}\left[\left(S_{a}^{(q) b}(P)+i \gamma_{5} \gamma_{\mu} A_{\mu, a}^{(q) b}(P)\right)\left(1+\frac{i P \gamma}{M}\right)\right]_{\alpha}{ }^{\beta}, \\
& \text { (Extrly.relat. comp.) } \quad V_{A}^{B}(P)=\frac{1}{2 \sqrt{2}}\left[\left(i \gamma_{5} P_{s, a}^{(E R) b}(P)+i \gamma_{\mu} V_{\mu, a}^{(E R) b}(P)\right)\left(1-\frac{i P \gamma}{M}\right)\right]_{\alpha}{ }^{\beta},
\end{aligned}
$$

where all vector and axial-vector mesons satisfy the Lorentz conditions, $P_{\mu} V_{\mu}(P)=$ $P_{\mu} A_{\mu}(P)=0$. Here it is to be noted that, in each type of the above members, the number of freedom counted both in the quark representation and in the meson representation is equal, as it should be $(2 \times 2=4$ and $1+3=4$, respectively). Also it may be amusing to note that each constituent in all the above members is in "parton-like motion," having the same 3-dimentional velocity as that of total mesons. (For example, in $\left.V(P), \mathbf{v}_{1,2}=\frac{\mathbf{p}^{(1,2)}}{p_{0}^{(1,2)}}=\frac{-\kappa_{1,2} \mathbf{P}_{M}}{-\kappa_{1,2} P_{M, 0}}=\mathbf{v}_{M} \cdot\right)$

In the heavy quarkonium $(Q \bar{Q})$ system both quarks and anti-quarks are possible to do, since $m_{Q}>\Lambda_{\text {conf }}$, only non-relativistic motions with positive energy. Accordingly the bi-spinor $U$ is considered to be applied to $Q \bar{Q}$ system as a covariant spin WF. In the heavy-light quark meson $Q \bar{q}(q \bar{Q})$ system the anti-quarks(quarks) make, since $m_{q} \ll \Lambda_{\text {conf }}$, relativistic motions both with positive and negative energies. Accordingly both the bispinors $U$ and $C(U$ and $D)$ are to be applied to the $Q \bar{q}(q \bar{Q})$ system, and in this system there is a possibility of existence of new composite scalar and axial-vector mesons(see Eq.(B)). In the light quark meson $q \bar{q}$-system both the quarks and anti-quarks make, since $m_{q} \ll \Lambda_{\text {conf }}$, relativistic motions both with positive and negative energies. Accordingly the (linear combinations to be specified shortly of) bispinors $U$ and $V$ are applied to the $q \bar{q}$-system, and in this system there is a possibility of existence of an extra(, in addition, to a normal) set of composite pseudo-scalar and vector mesons.

\section{Light-Quark Meson Systems and Chiral Symmetry}

A) [Charge conjugation] properties of the bi-spinors and, correspondingly, of the composite mesons are derived from those of quarks as follows:

$\Psi_{A}^{(+) B}(P, x) \quad\left(\quad \approx\left\langle 0\left|\psi_{A}\left(x_{1}\right) \bar{\psi}^{B}\left(x_{2}\right)\right| M\right\rangle\right) \rightarrow \Psi_{A}^{c,(+) B}(P, x)\left(\approx\left\langle 0\left|\psi_{A}\left(x_{1}\right) \bar{\psi}^{B}\left(x_{2}\right)\right| M^{c}\right\rangle\right)$ 


$$
\begin{aligned}
& =\left(C^{-1}\right)_{A A^{\prime}}{ }^{t} \Psi^{(+)}(P,-x)^{A^{\prime}}{ }_{B^{\prime}} C^{B^{\prime} B}, \quad\left(C C^{\dagger}=1, C \gamma_{\mu} C^{-1}=-{ }^{t} \gamma_{\mu}\right) ; \\
P_{s, a}^{(N R) b} & \leftrightarrow P_{s, b}^{(N R) a}, V_{\mu, a}^{(N R) b} \leftrightarrow-V_{\mu, b}^{(N R) a} ; S_{a}^{(R \bar{q}) b} \leftrightarrow S_{b}^{(R q) a}, A_{\mu, a}^{(R \bar{q}) b} \leftrightarrow A_{\mu, b}^{(R q) a} \\
P_{s, a}^{(E R) b} & \leftrightarrow P_{s, b}^{(E R) a}, V_{\mu, a}^{(E R) b} \leftrightarrow-V_{\mu, b}^{(E R) a} .
\end{aligned}
$$

B)[Chiral transformation] properties of composite mesons are also derived straightforwardly from those of the bi-spinors

$\psi_{A}^{B}(P, x) \rightarrow\left[e^{i \alpha^{i} \frac{\lambda^{i}}{2} \gamma_{5}} \psi(P, x) e^{i \alpha^{i} \frac{\lambda^{i}}{2} \gamma_{5}}\right]_{A}^{B}$.

\section{C) [Light-quark meson system- "chiral SU(6) multiplet"]}

The quark representation applying to the light-quark mesons is obtained by the linear transformation of the bi-Dirac spinors given in $\S 3$ as follows:

$\begin{aligned} U_{P_{s}, \alpha}^{(N, E) \beta} & \equiv 1 / \sqrt{2}\left(U_{P_{s}} \pm V_{P_{s}}\right)_{\alpha}{ }^{\beta}=i / 2\left[\gamma_{5}(1, i v \gamma)\right]_{\alpha}{ }^{\beta} ; \quad P_{s}^{(N, E)} ; C=(+,+) \\ C_{S, \alpha}^{(N, E) \beta} & \equiv(1, i) / \sqrt{2}\left(D_{S} \pm C_{S}\right)_{\alpha}{ }^{\beta}=1 / 2[(1,-v \gamma)]_{\alpha}{ }^{\beta} ; \quad S^{(N, E)} ; C=(+,-) \\ U_{V, \alpha}^{(N, E) \beta} & \equiv 1 / \sqrt{2}\left(U_{V} \pm V_{V}\right)_{\alpha}{ }^{\beta}=i / 2\left[\left(\tilde{\gamma}_{\mu},-\sigma_{\mu \nu} v_{\nu}\right)\right]_{\alpha}{ }^{\beta} ; \quad V^{(N, E)} ; C=(-,-) \\ C_{A, \alpha}^{(N, E) \beta} & \equiv(1, i) / \sqrt{2}\left(D_{A} \pm C_{A}\right)_{\alpha}{ }^{\beta}=i / 2\left[\gamma_{5}\left(\tilde{\gamma}_{\mu},-i \sigma_{\mu \nu} v_{\nu}\right)\right]_{\alpha}{ }^{\beta} ; \quad A^{(N, E)} ; C=(+,-)\end{aligned}$

$\left(v_{\mu} \equiv P_{\mu} / M, \tilde{\gamma}_{\mu} v_{\mu} \equiv 0\right.$; and $U_{P_{s}}$ denotes the coefficient bi-spinors of $P_{s}$ and so on ), where we have given also the charge-conjugation parity of the corresponding (hidden flavor) composite mesons. The chiral transformation properties of the new bi-spinors (for $\left.U_{L}(1) \times U_{R}(1)\right)$ are easily seen to be similar as the conventional ones as

$$
1 \leftrightarrow i \gamma_{5}, \quad-v \gamma \leftrightarrow-\gamma_{5} v \gamma, \quad i \gamma_{5} \tilde{\gamma}_{\mu} \leftrightarrow i \tilde{\gamma}_{\mu}, \quad-i \sigma_{\mu \nu} v_{\nu} \leftrightarrow-\gamma_{5} \sigma_{\mu \nu} v_{\nu}
$$

\section{D) ["Local chiral SU(6) field"]}

Extending our considerations to include the (-)-frequency part, we are led to a unified expression of what to be called, Local Chiral SU(6) field, as

$$
\begin{aligned}
\Psi_{A}^{B}(X)= & \Psi_{A}^{(N) B}(X)+\Psi_{A}^{(E) B}(X) \\
\Psi_{A}^{(N) B}= & 1 / 2\left[i \gamma_{5 \alpha}{ }^{\beta} P_{s, a}^{(N) b}+i \tilde{\gamma}_{\mu, \alpha}{ }^{\beta} V_{\mu, a}^{(N) b}+1_{a} l p h a^{\beta} S_{a}^{(N) b}+\left(i \gamma_{5} \tilde{\gamma}_{\mu}\right)_{\alpha}{ }^{\beta} A_{\mu, a}^{(N) b}\right] \\
\Psi_{A}^{(E) B}= & 1 / 2\left[\left(i \gamma_{5} \gamma_{\mu}\right)_{\alpha}{ }^{\beta} M^{-1} \partial_{\mu} P_{s, a}^{(E) b}+\left(\sigma_{\mu \nu}\right)_{\alpha}{ }^{\beta} M^{-1} \partial_{\mu} V_{\nu, a}^{(E) b}\right. \\
& \left.+i \gamma_{\mu, \alpha}{ }^{\beta} M^{-1} \partial_{\mu} S_{a}^{(E) b}+\left(i \gamma_{5} \sigma_{\mu \nu}\right)_{\alpha}{ }^{\beta} M^{-1} \partial_{\mu} A_{\nu, a}^{(E) b}\right] .
\end{aligned}
$$

\section{Concluding remarks}

In this talk we have pointed out a possibility of existence of the new composite meson multiplets, when both or either constituent quark-excitons have "negative" energies and momenta. Here we should like to point out that the chiral symmetry of QCD guarantees this to be the case, and there exist some candidates (to be discussed elsewhere) for these new multiplets.

\section{REFERENCES}

1. S. Ishida, M. Y. Ishida and M. Oda, Prog. Theor. Phys. 93, 939 (1995).

2. H. Yukawa, Phys. Rev. 91, 415 and 416 (1953).

3. S. Ishida, Prog. Theor. Phys. 46, 1570 and 1950 (1971). 